\title{
Epigenetics: biology's quantum mechanics
}

\author{
Richard A. Jorgensen* \\ Laboratorio (LANGEBIO) Nacional de Genómica para la Biodiversidad, Centro (CINVESTAV) de Investigación y Estudios Avanzados, Irapuato, Guanajuato, México
}

Edited by:

Ingo Schubert, Leibniz Institute of Plant Genetics and Crop Plant Research,

Germany

\section{Reviewed by:}

Peter James Shaw, John Innes Centre, UK

Andreas Houben, Leibniz Institute for Plant Genetics and Crop Plant

Research, Germany

Michael Florian Mette, Leibniz Institute of Plant Genetics and Crop Plant

Research, Germany

\section{*Correspondence:}

Richard A. Jorgensen, Laboratorio

Nacional de Genómica para la

Biodiversidad, Centro de Investigación

y Estudios Avanzados, Km 9.6

Libramiento Norte Carretera a León,

36821 Irapuato, Guanajuato, Mexico.

e-mail: rajorgensen@langebio.

cinvestav.mx
The perspective presented here is that modern genetics is at a similar stage of development as were early formulations of quantum mechanics theory in the 1920s and that in 2010 we are at the dawn of a new revolution in genetics that promises to enrich and deepen our understanding of the gene and the genome. The interrelationships and interdependence of two views of the gene - the molecular biological view and the epigenetic view - are explored, and it is argued that the classical molecular biological view is incomplete without incorporation of the epigenetic perspective and that in a sense the molecular biological view has been evolving to include the epigenetic view. Intriguingly, this evolution of the molecular view toward the broader and more inclusive epigenetic view of the gene has an intriguing, if not precise, parallel in the evolution of concepts of atomic physics from Newtonian mechanics to quantum mechanics that are interesting to consider.

Keywords: aperiodic crystal, paragenetics, parachromatin, transgenerational inheritance, histone code

\section{PHYSICS AND THE MOLECULAR UNDERSTANDING OF THE GENE}

In a classic little book, "What is Life?" the great theoretical physicist Erwin Schrödinger considered one of biology's central questions: how can "a living organism be accounted for by physics and chemistry?" (Schrödinger, 1944). In attempting to answer this question, Schrödinger rightly focused on the centrality of the gene, and thus on the necessity of understanding the nature of mutations given that they are essential for identifying and investigating the nature of genes. Because the chemical nature of the gene was unknown the paper by Avery et al. (1944) demonstrating that DNA was the genetic substance was published while "What is Life?" was still in press -Schrödinger drew on Max Delbrück's atomic physics-based model of genetic mutation and the structure of the gene (TimofeeffRessovsky et al., 1935) suggesting that the gene is a linear onedimensional crystal. Schrödinger referred to it as an "aperiodic crystal," i.e., one whose elements do not repeat in a periodic way as in common crystals.

Although Schrödinger's little book was not universally well received, especially by biologists, even a critic like Linus Pauling was willing to conclude that Schrödinger's formulation of the theory of wave mechanics in 1925, for which he received the Nobel Prize in 1933, was "basically responsible for modern biology" because it developed largely on the basis of the new understanding of chemistry that quantum mechanics made possible (Pauling, 1987; cited in Dronamraju, 1999).

Quantum mechanics was certainly also at the core of Schrödinger's speculations on the nature of the gene. "What is Life?" offered a fresh perspective on biology that inspired the new "molecular biologists," many of whom had been trained as physical scientists, especially crystallographers and physical chemists (such as Max Delbrück, Linus Pauling, Francis Crick, and Matthew Meselson, to name just a few). This was the dawn of the great molecular biology revolution that led to a vast increase in our understanding of genes and genomes and established the fundamental nature of the gene as a nucleic acid molecule comprised of a string of distinct nucleotide bases whose sequence normally specifies a gene product which can effect or influence the expression of phenotype during growth and development.

\section{THE EARLY EPIGENETIC PERSPECTIVE AND THE CONCEPT OF "PARACHROMATIN"}

Interestingly, a complementary way of thinking about the nature of the gene that came to be associated with the term "epigenetics" also had its origins in the 1930s and 1940s. The epigenetic perspective pointed toward a more complicated reality for the nature of genes that was largely sidelined for several decades by the molecular biology revolution - for good reason, because it was molecular biology that would have to develop the tools and approaches to eventually understand the molecular basis of the epigenetic perspective. Thus, the two perspectives existed in parallel, with little cross-talk, simply because molecular biology had to develop and mature to the point that it could consider and address the epigenetic perspective.

As first noted by Brink (1960), the epigenetic perspective was presaged by none other than Morgan (1934), when he noted the possibility that "the genes also are building up more and more, or changing in some way as development proceeds, in response to that part of the protoplasm in which they come to lie." This view of the gene as dynamically changing in development "without losing its fundamental properties" (Morgan, 1934) has been undergoing 
a renaissance in recent years, as it has become increasingly clear that the DNA-focused understanding of the gene that had revolutionized genetics and biology is actually incomplete without its complement, the "chromatin"-centered perspective of epigenetics.

\section{PARACHROMATIN: CHROMOSOMALLY BASED EPIGENETIC STATES}

The chromatin-centered perspective on the gene was first elaborated 50 years ago by R. Alexander Brink. In a classic paper, Brink (1960) inferred that eukaryotic chromosomes not only have a genetic function, but also possess what he termed a "paragenetic" function. He proposed that a genetic locus was thus comprised of two types of chromatin, which he termed "orthochromatin" and "parachromatin." Brink defined orthochromatin as the substance at a locus that remains qualitatively constant in all nuclei of an individual and is presumably comprised of the DNA at a given locus. Parachromatin, on the other hand, Brink proposed to be comprised of alternative states of chromatin that can be altered by factors arising in development or in response to the environment, and that these states can adopt a succession of mitotically transmissible states, together reflecting the progressive history of a cellular lineage. Brink explicitly noted that the concepts of euchromatin and heterochromatin are fundamentally different than the concepts of orthochromatin and parachromatin, especially in that the locations of the former pair are mutually exclusive, whereas the latter pair are co-located intimately at the same genetic locus.

Brink based his hypothesis of a paragenetic function for chromosomes on observations that he and two other maize geneticists, Barbara McClintock and Edward Coe, made in the 1950s, which demonstrated the existence of two types of genetic variations, classical mutations and what he termed "paramutations." Classical mutations are undirected and discrete, whereas "paramutations," in the broadest use of the term (Brink, 1960), are directed, for instance by particular "paramutagenic" alleles, by development, or by the environment. Most "paramutations" are also typically reversible and changeable, often exhibiting a series of quantitatively or qualitatively varying states. Brink argued that although paramutations violate Mendel's laws, their occurrence implies the existence of processes by which genes change in development and supports Morgan's contention that the genes might change in development without losing their fundamental (i.e., genetic) properties.

What is "parachromatin" in physical terms? Brink's definition of parachromatin allowed great latitude in the nature, composition, and dynamic behaviors of "parachromatin." In modern terms, we would include not only histone proteins and their various modification states, but also the many, diverse chromatin proteins and complexes that "remodel" chromatin into new "states" that may be comprised of conformational alterations, covalent modifications, and/or changes in molecular composition. Explicitly included in such chromatin "states" would be not only proteins, but also RNA molecules of various types, including but not limited to siRNAs produced by RNA interference-related chromatin complexes. Thus, the modern view of chromatin at a given locus is one of a highly dynamic entity that fits well with Brink's concept of parachromatin. Even though Brink had no understanding of the molecular nature of chromatin, his hypothesis was flexible enough to fully encompass our modern understanding of chromatin.
Brink's concept of "parachromatin" offered a broad general perspective on the nature, behaviors, and functions of genes in eukaryotes. But it was impossible in 1960 for Brink to specify any molecular details or mechanisms that comprise parachromatin. Only 1 year later, Jacob and Monod (1961) offered a simple, straightforward molecular biological model of the control of gene expression in prokaryotes that revolutionized thinking about how genes were expressed and regulated and that seemed to have the potential to account for the gene regulation in complex eukaryotes as well. Parachromatin was largely forgotten for decades, as molecular biologists and geneticists explored the Jacob-Monod model of gene regulation in great detail, first in prokaryotes and then in eukaryotes.

Eventually, however, it came to be recognized that simple models based on transcription factors binding to specific DNA sequences were insufficient on their own to explain the control of gene regulation in complex eukaryotes. Molecular tools and approaches were developed that could begin to open a window on the higher order complexities of eukaryotic gene regulation. Chromatin was gradually accepted, first as a mediator or modulator of transcription complex formation, and then as an active, dynamic participant in the entire transcription process. When the concept of the "histone code" or the "histone language" was developed around 2000, molecular biology was finally beginning to catch up to Brink's (1960) concept of parachromatin. Of course, we are still very far from being able to say that we fully understand the molecular basis of parachromatin, but I have little doubt that we have begun to open the door to a vast realm of possibilities waiting to be explored and to be defined molecularly and mechanistically.

\section{THE GENE AS A "FIELD OF POSSIBILITIES?"}

The evolution from Mendelian genetics toward what we might call "molecular epigenetics" via molecular biology's description of the gene as a nucleic acid molecule has an intriguing and perhaps instructive parallel in the evolution of physics from Newtonian mechanics toward quantum mechanics via the "planetary model" of the atom in which electrons orbit the nucleus. The original concept of the atom, the fundamental entity of Newtonian mechanics, had held that the atom was an indivisible particle. This concept was transformed twice - first into the planetary model and then by quantum mechanics which redefined the atom as a field of probabilities" of subatomic particles existing in four dimensions (three spatial dimensions and time).

Similarly, the original concept of the gene, the fundamental entity of Mendelian genetics, was that it was also particulate and indivisible, a concept that has also been transformed twice - first through the prism of genetics and molecular biology into the concept of a gene as a nucleotide sequence, divisible by recombination, and now again by molecular biology, looking through the prism of epigenetics, into what might be referred to as a "field of possibilities" of alternative chromatin states centered on a particular nucleic acid sequence, i.e., parachromatin, to use Brink's terminology. As mentioned previously, alternative parachromatin states are assumed to be based on diverse chromatin proteins, RNAs, and complexes that can exist in various conformational and covalent modification states that are adopted during growth and development and can differ among loci. 
In quantum mechanics, subatomic particles may be viewed in two very different ways, i.e., as both waves and particles; likewise, paramutations may be viewed both as gene expression states and as mitotically, and in some cases, meiotically heritable states. Classical genetics and then molecular biology established the macro-framework of genetics in the same way that Newtonian mechanics and then the planetary model of the atom established the macro-framework of physics. Quantum mechanics ultimately led to the idea of wave-particle duality and the description of positions of subatomic particles as a "field of probabilities" that determine the chemical properties of a given atom. Similarly, Brink introduced the idea of a genetic-paragenetic duality of genetic loci in which a genetic entity (DNA) and a paragenetic entity (chromatin), both present at each genetic locus, are complementary and interdependent partners which we might describe as a "field of possibilities" that determine the expression states of a given locus.

In drawing this parallel, I am not trying to suggest that the classical, molecular biological view of genetics is neither correct nor useful, any more than Newtonian mechanics or the "planetary" model of the atom is neither correct nor useful. Rather, I am simply suggesting that each represents early, incomplete descriptions of reality that required important modification and enrichment before we could fully understand the nature of the genetical and physical worlds, respectively. However, we ought not try to take the analogy too far because at some level of detail it most likely will break down. Nonetheless, we can explore how far the analogy might or might not extend.

\section{UNCERTAINTY IN PHYSICS AND BIOLOGY}

In the twentieth century, physics evolved beyond a deterministic view of the universe postulating that the future of the universe in theory could be extrapolated from the laws of physics if only one could obtain complete knowledge of the positions, directions, and velocities of movement of all particles in the universe. Quantum mechanics, especially in Heisenberg's uncertainty principle raised fundamental questions that challenged the possibility of precise knowledge of the future: For instance, the number of times per second that atoms in a lump of uranium will undergo radioactive decay is known with precision; however, why and when any particular atom will decay is unpredictable by modern physics.

Similarly, although geneticists can measure mutation frequency in a particular system under specific conditions, the timing of a particular nucleotide substitution (or any other mutational event) is unpredictable. Only the likelihood of the mutation can be known. Thus, from an evolutionary genetic perspective, biology is no more deterministic than is physics, as Tautz (2000) has analyzed in terms of population genetic theory. Tautz argued that the selective fitness of an advantageous mutation in a population and time comprise a "canonically conjugated" pair of variables analogous to such pairs of physical variables, such as the location and momentum of a particle, or the energy of a particle and the time at which it was measured. He showed that uncertainty is largest at low allelic frequency and when the selective advantage is small. The specific trajectory of such an allele in a population of finite size is well known in population genetics to be unpredictable, and only probabilities for different trajectories can be determined.
With the advent of genomics, it is theoretically possible to know with absolute certainty the sequence of a region of chromosome carrying a gene and even the sequence of an entire chromosome. However, as Stadler (1954) noted, it is not trivial to precisely locate a gene, i.e., it cannot "be shown to be delimited from neighboring genes by definite boundaries.” This conclusion follows from Stadler's definition of the gene: "operationally, the gene can be defined only as the smallest segment of the gene-string that can be shown to be consistently associated with the occurrence of a specific genetic effect." In modern terms, knowing the complete sequence of a chromosome does not allow us to precisely determine all of the "many interdependent elements of a gene, including all those elements in cis that are necessary for the normal operation of a given gene" that is associated with a specific genetic effect (Jorgensen, 2010). In addition, the expression and selective value of a gene in nature may often be dependent on the environment encountered by the organism, perhaps making it impossible to precisely identify the boundaries of a gene.

Distinct from quantum mechanics, it is also important to recognize the relevance to biology of complexity theory, which has identified another type of uncertainty in physics, resulting from sensitive dependence on initial conditions such that relatively simple Newtonian systems may exhibit unpredictable "chaotic" behaviors due to the impracticality of knowing initial conditions precisely enough.

Similarly, it should be evident that knowing all alternative epigenetic states of a given gene in all environments may be unachievable in any practical sense. This is essentially no different than what is postulated in complexity theory, namely, that it is impractical to know with enough precision the locations and movements of all particles of a system in order to predict future behavior with any certainty, at least in certain systems and under certain conditions. Perhaps then, the most we can hope to achieve is to determine or estimate the "field of possibilities" that comprise the orthochromatin and parachromatin components of each gene.

\section{CONCLUDING COMMENTS}

The marriage of epigenetics and molecular biology promises to deepen and revolutionize our understanding of the fundamental nature of the gene, allowing us to see deeply into its "field of possibilities." It is going to be very interesting to learn what degree of knowledge of the "field of possibilities" that comprise a gene we will be able to obtain. Though I will be happy to be proved wrong, my strong suspicion is that while we will be able to learn a lot about a given gene, we can never know what we do not know about it, i.e., its complete "field of possibilities" will be not be determinable simply because it will be impossible to anticipate for every gene every circumstance that an organism may encounter.

Finally, given the rapidly increasing interest in the phenomenon of "transgenerational" (meiotic) inheritance of epigenetic and paragenetic states and their possible evolutionary consequences, it is also interesting to consider that epigenetics may also revolutionize our understanding of biological evolution (perhaps similar to how understanding of atomic particles continues to revolutionize our understanding of the evolution of the universe). Biological evolution fundamentally depends on the existence and nature of genetic variations. Many biologists, 
especially plant biologists, have long postulated that information may sometimes flow "backward" from the environment to the gene via epigenetic impositions on the genome (altering states of Brink's "parachromatin") and have suggested the possibility for the generation of novel genetic variations which, in the words of Barbara McClintock, might "vary according the nature of the challenge to be met" (McClintock, 1978). This seemingly radical suggestion (which was not Lamarckian, but rather a modified Darwinian mechanism) led to a great deal of criticism and

\section{REFERENCES}

Avery, O. T., MacLeod, C. M., and McCarty, M. (1944). Studies on the chemical nature of the substance inducing transformation of pneumococcal types: induction of transformation by a desoxyribonucleic acid fraction isolated from pneumococcus type III. J. Exp. Med. 79, 137-158.

Brink, R. A. (1960). Paramutation and chromosome organization. Q. Rev. Biol. 35, 120-137.

Dronamraju, K. R. (1999). Erwin Schrödinger and the origins of molecular biology. Genetics 153, 1071-1076.

Jacob, F., and Monod, J. (1961). Genetic regulatory mechanisms in the synthesis of proteins. J. Mol. Biol. 3 , 318-356.
Jorgensen, R. A. (2004). Restructuring the genome in response to adaptive challenge: McClintock's bold conjecture revisited. Cold Spring Harb. Symp. Quant. Biol. 69, 349-354.

Jorgensen, R. A. (2010). Of genes and genomes: challenges for the twentyfirst century. Front. Plant Sci. 1:1. doi: 10.3389/fpls.2010.00001

McClintock, B. (1978). Mechanisms that rapidly reorganize the genome. Stadler Genet. Symp. 10, 25.

Morgan, T. H. (1934). Embryology and Genetics. New York: Columbia University Press, 234.

Pauling, L. (1987). "Schrödinger's contribution to chemistry and biology," in Schrödinger: Centenary Celebration of a Polymath, ed. C. W. Kilmister

misunderstanding of McClintock - unfair criticism, as I have argued previously; rather than repeat that discussion here, I would refer the interested reader to Jorgensen (2004).

\section{ACKNOWLEDGMENTS}

The author would like to thank Jean-Philippe Vielle-Calzada for his helpful comments on the manuscript, Gane Ka-Sha Wong for bringing Tautz (2000) to my attention, as well as the three reviewers, whose comments also helped to improve the presentation substantially.

(Cambridge: Cambridge University Press), 225-233.

Schrödinger, E. (1944). What is Life? Cambridge: Cambridge University Press Stadler, E. J. (1954). The gene. Science 120, 811-819.

Tautz, D. (2000). A genetic uncertainty principle. Trends Genet. 16, 475-477.

Timofeeff-Ressovsky, N. W., Zimmer, K. G., and Delbrück, M. (1935). Ueber die Natur der Genmutation und der Genstruktur. Nachr. Ges. Wiss. Goettingen, Fachgr. 6, N.F. 1, 13, 190-245.

Conflict of Interest Statement: The author declares that the research was conducted in the absence of any commercial or financial relationships that could be construed as a potential conflict of interest.

Received: 01 February 2011; accepted: 31 March 2011;published online: 12 April 2011. Citation: Jorgensen RA (2011) Epigenetics: biology's quantum mechanics. Front. Plant Sci. 2:10. doi: 10.3389/fpls.2011.00010

This article was submitted to Frontiers in Plant Genetics and Genomics, a specialty of Frontiers in Plant Science.

Copyright (c) 2011 Jorgensen. This is an open-access article subject to a nonexclusive license between the authors and Frontiers Media SA, which permits use, distribution and reproduction in other forums, provided the original authors and source are credited and other Frontiers conditions are complied with. 